\title{
La escritura académica y su evaluación (una experiencia con estudiantes de ingeniería a partir de un tema de geometría analítica)
}

\author{
Vicente Messina ${ }^{1}$, Gloria Cittadini' ${ }^{2}$ Carlos Pano $^{3}$ \\ vrmessi@gmail.com
}

\section{Resumen}

En este trabajo narramos una actividad de escritura académica realizada por estudiantes de ingeniería y su evaluación. Para ello elegimos un capítulo de un texto universitario que trata sobre secciones cónicas. Describimos el contexto y el trabajo de los alumnos. Caracterizamos la noción de trabajo práctico y su aplicación a este caso. Señalamos el concepto de evaluación seguido. Especificamos los aspectos de un escrito académico en el marco de alfabetización académica y su evaluación en los escritos de los alumnos. Presentamos los resultados. El desarrollo de la actividad estuvo mediado por el aula virtual. Sostenemos que la práctica de la escritura académica aporta a una mejor formación de los ingenieros.

Palabras clave: alfabetización académica - escritura académica - evaluación - geometría analítica - entropía.

\footnotetext{
1 Licenciado en Enseñanza de las Ciencias por la Universidad Nacional de General San Martín Especialista y maestrando en Metodología de la Investigación Científica por la Universidad Nacional de Lanús. Profesor Adjunto en Álgebra y Geometría Analítica e investigador en la Facultad Regional Buenos Aires de la Universidad Tecnológica Nacional.

2 Licenciada en Tecnológia Educativa. Profesora Titular Ordinaria en Álgebra y Geometría Analítica e investigadora en la Facultad Regional Buenos Aires de la Universidad Tecnológica Nacional.

3 Licenciado en Ciencias Matemáticas. Profesor Titular Ordinario de Probabilidad y Estadística e investigador en la Facultad Regional Buenos Aires de la Universidad Tecnológica Nacional. Profesor Titular de Estadística en la Facultad de Psicología de la Universidad de Buenos Aires.
} 


\section{Abstract}

This work is devoted to narrate the academic writing activity done by engineering students, as its assessment. Therefore, the chosen text was about conic sections. The context where the experience was realized and the student's work was described. The concept of the task, as well as its application was characterized. The followed assessment concept was indicated. Then, the academic writing's aspects were evaluated in the academic literacy framework. The results were presented. The virtual campus made possible the activity's development. From the work it is concluded that the practice of academic writing contributes to a better engineer's formation.

Keywords: academic literacy - academic writing - evaluation - analytic geometry - entropy.

\section{Introducción}

Todas las instituciones educativas (desde el jardín de infantes hasta la Universidad) son responsables de que las personas comprendan mensajes cada vez más complejos de la mejor forma posible. Es por eso que la alfabetización no debería ser considerada privativa de la escuela primaria sino establecerse como un proceso de aprendizaje permanente, en especial porque el vínculo de los adultos con la lengua escrita es mayormente de resistencia a su uso y de dificultad para abordarla de manera placentera y correcta (Castedo, 1995).

Como docentes de Álgebra y Geometría Analítica para carreras de Ingeniería, y en el marco del Proyecto de Investigación Alfabetización académica en entornos virtuales de aprendizaje y evaluación innovadora con función pedagógica (2013), en el cual participamos, hemos realizado, con estudiantes de la materia, una experiencia de escritura académica. El objetivo fue realizar una actividad de alfabetización académica en un entorno virtual de aprendizaje. Este trabajo reporta los resultados obtenidos.

La escritura de los alumnos en la universidad está ligada a la lectura y a la comunicación oral. Los estudiantes producen escritos a partir de la lectura de la bibliografía que leen, de la escucha de lo trasmitido por los docentes y de las interacciones que ocurren en el aula entre ellos y con el profesor. En el caso de la experiencia, los escritos que les pedimos se sustentaron principalmente con la lectura de un capítulo de libro recomendado. También pudieron rescatar ideas del foro virtual al que pudieron acceder.

La escritura académica requiere de la lectura reflexiva del texto elegido, no para reproducirlo sino para iniciar el desarrollo de la propia argumentación. Se trata de un trabajo mental de comprender y ordenar las ideas expuestas en el texto y de cotejarlas con las propias. La escritura académica precisa planificación, es decir, formulación de objetivos que pueden modificarse mientras se escribe en virtud de la aparición de nuevas ideas. Necesita transformación, "transformación supone el acceso a la memoria semántica, encontrar la palabra que expresa la idea, colocar la palabra en la frase y leer lo que 
Vicente Messina , Gloria Cittadini y Carlos Pano / La escritura académica y [...] 137 está saliendo" (Bruning, Schraw, Norby y Ronning, 2005, p. 345). Necesita también de revisión para hacer las correcciones y modificaciones que se consideren apropiadas, lo que implica la reorganización y reescritura de lo hecho.

La escritura académica es un modo de apropiarse y transformar el conocimiento que favorece el análisis crítico sobre el propio saber. La palabra escrita no sólo expresa el pensamiento sino que su producción lo desarrolla y le da consistencia. Para agregarle valor, resulta preciso escribir con conciencia retórica, esto es, anticipar el efecto del propio texto sobre el lector. Formar a los estudiantes en escritura académica es una manera de alfabetizar en la Universidad. Su práctica es una actividad cognitiva donde el estudiante construye un nuevo conocimiento, combinando lo que ya sabe sobre un tema con el conocimiento que posee sobre los procesos del discurso y sus objetivos.

La Alfabetización Académica, de acuerdo con Thaiss, Bräuer, Carlino, Grabocsik-Willians y Sinha (2012) y Carlino (2005), permite apropiarse de algunas de las formas de razonamiento instituidas en la cultura discursiva de una disciplina científica o tecnológica. No es un enfoque que pretende remediar deficiencias en la formación previa, sino que implica que cada una de las cátedras esté dispuesta a abrir las puertas de la disciplina que enseña. Abrir las puertas de Álgebra y Geometría Analítica nos encamina en la dirección de mejorar los aprendizajes de la asignatura.

\section{Contexto}

La actividad que describimos se desarrolló en un curso de Álgebra y Geometría Analítica, asignatura que se cursa en forma anual, con una carga horaria de cinco horas semanales. El curso contó con 61 alumnos que comenzaban su formación universitaria, habían cursado y aprobado el Seminario Universitario de Ingreso que se imparte en la Facultad durante los meses de febrero y marzo. Ellos cursaban por primera vez materias del primer nivel de la Facultad Regional Buenos Aires de la Universidad Tecnológica Nacional. La Universidad Tecnológica Nacional fue creada en 1959 y desde ese año han egresado más de 30.000 profesionales de las distintas carreras de grado. Una de sus características principales es la de ser la única Universidad del país en priorizar la enseñanza y el aprendizaje de las distintas ingenierías. Otra es su carácter federal, dado que está integrada por 29 Facultades Regionales que se encuentran distribuidas por todo el país. Esto implica que los sistemas productivos regionales están en permanente relación con la Universidad, generando un intercambio académico a nivel nacional. El alumnado que cursa las distintas ingenierías en todas estas Regionales es aproximadamente de 70.000 alumnos que equivale a más del $50 \%$ de todos los estudiantes de ingeniería del país. A pesar de esta masividad, la Universidad considera importante que la razón entre la cantidad de alumnos y la cantidad de docentes por curso permita una relación docente-alumno personalizada. Desde su origen está comprendida en la concepción asociativa universidad-trabajo. En lo concreto, esto se traduce en horarios de clase compatibles con la actividad laboral, clases teórico-prácticas de tipo seminario o taller. Respecto a la asignatura Álgebra y Geometría Analítica, cabe aclarar que ésta es común a todas las especialidades de las carreras de Ingeniería, junto con otras asignaturas de ciencias básicas que se imparten en el mismo nivel. El contenido medular del curso consiste en una introducción al Álgebra Lineal y al tratamiento de la Geometría Analítica desde un enfoque vectorial. 


\section{8/ Perspectivas Metodológicas /19/Vol. II /Año 2017}

En el curso donde fue desarrollada la experiencia, las clases presenciales abordaron los temas propios del programa. La actividad de escritura académica se gestionó a través del aula virtual, allí estuvieron instalados los materiales y allí también los alumnos dejaron sus producciones. Las consultas se resolvieron a través del foro del aula virtual. Los alumnos contaron con computadora o facilidad para utilizar una máquina. Pudieron acceder a internet tanto en la Universidad como en sus domicilios, así como también al aula virtual asignada al curso. La clase estuvo a cargo de dos profesores: la profesora titular y el jefe de trabajos prácticos. Los dos docentes participaron en cada clase presencial y tuvieron a su cargo el desarrollo de la experiencia que describimos en el marco del proyecto antes mencionado.

\section{Sobre los trabajos prácticos}

Los trabajos prácticos son un instrumento cuyo objetivo es relacionar la teoría con la práctica y que exige a los alumnos comprensión y análisis de los conceptos puestos en juego por el docente. De acuerdo con Steiman (2008), "Consideramos al trabajo práctico como una propuesta de interpretación y fundamentación teórica que parte de una situación problemática global que contextualiza a cada una de las tareas a realizar" (Steiman, 2008, pp 85-86). Éste análisis y comprensión implica que los alumnos deban interactuar con sus conocimientos previos, con los contenidos explicados, con la lectura de los textos sugeridos; les exige saber qué es lo que saben, pero teniendo simultaneamente en cuenta conciencia de lo que no saben. Para la resolución del trabajo práctico deben usar en forma integrada sus conocimientos y habilidades adquiridas, apelando en algunos casos a la creatividad. Los trabajos prácticos se pueden resolver de manera individual o grupal, en el aula o fuera de ella, o en un contexto virtual. Cualquiera sea el caso, los mismos contribuyen a la construcción de los conocimientos específicos de la asignatura.

Según Doyle (1981), la forma de pensar del alumno está condicionada por las tareas que se le solicitan. Los docentes guían el aprendizaje de los alumnos al diseñar e implementar tareas académicas; en el desarrollo de estos diseños, el profesor reflexiona sobre los contenidos, la organización, la secuencia de las actividades, su adecuación al curso y puede prever las posibles interacciones en el aula. Doyle (1982) señala además la tipología de tareas académicas de acuerdo con los procesos cognitivos: tareas de memoria, actividades de rutina o procedimientos, tareas de comprensión, tareas de opinión. A nuestro entender, se deberían agregar tareas de creación. Es posible diseñar trabajos prácticos en los cuales los alumnos deban hacer análisis e interpretaciones cada vez más profundos. Esta profundización requiere demandas cognitivas que la acompañen.

Los trabajos prácticos usualmente propuestos en la asignatura Álgebra y Geometría Analítica están pensados para que el alumno desarrolle un cierto entrenamiento en resolver ejercicios, utilizando la fórmula adecuada o siguiendo los pasos convenientes para llegar a la respuesta correcta. Estos trabajos prácticos se sirven del lenguaje de la asignatura, de modo tal que obligan al alumno a tratar de comprenderlo. No ofrecen posibilidades de profundizar, pues se limitan a los contenidos que están estipulados en el programa y a los objetivos de la asignatura. No contienen ejercicios integradores y 
Vicente Messina, Gloria Cittadini y Carlos Pano / La escritura académica y [...] 139 muchos de los ejercicios están destinados a que el estudiante memorice fórmulas, procedimientos y propiedades. No están elaborados para que el alumno trabaje con sus pares. Cuando el alumno se encuentra frente a un ejercicio, intuye que debe descubrir cuál es el procedimiento correcto para encontrar la respuesta.

\section{1. El diseño de los trabajos prácticos para la experiencia}

Para el diseńo de los trabajos prácticos, preparamos una serie de preguntas y ejercicios que el alumno pudiera responder a partir de la lectura del capítulo elegido. Elaboramos cinco trabajos prácticos. En cada uno de estos se indica cuál es la sección del capítulo que abarca los contenidos necesarios para la realización del trabajo, los objetivos a lograr y las pautas de lectura y escritura que el alumno debe seguir para el desarrollo del escrito pedido.

A través de estas actividades, buscamos que el alumno pueda precisar por escrito los conceptos leídos, emplear los procedimientos expuestos en la resolución de los ejercicios, utilizar el lenguaje formal de las matemáticas y valorar su rigurosidad. También buscamos que pueda apelar a representaciones gráficas utilizando el software que le indicamos. Asimismo, nos interesa que pueda adquirir un manejo adecuado del vocabulario del tema, dándole a los términos el significado que les corresponde en el espacio contextual de las cónicas.

\subsection{Texto propuesto a los alumnos para su lectura}

El texto elegido es el capítulo 3: "Secciones Cónicas", del libro Nociones de Geometría Analítica y Álgebra Lineal, escrito por Kozak, A., Pastorelli, S., Vardanega (2007). La elección de este capítulo se debe al tratamiento que los autores hacen del tema. Este tratamiento nos permite ubicar la mencionada sección del libro al comienzo de la cursada de Algebra y Geometría Analítica y de forma paralela al curso presencial. Circunstancia que podía facilitar la realización de la experiencia.

El libro seleccionado está en la bibliografía de la asignatura. Tiene la particularidad de que los autores son docentes de distintas Regionales de la Universidad Tecnológica Nacional. Sigue los temas del programa, la presentación es clara en un nivel adecuado para los alumnos que comienzan en una carrera de ingeniería. Cada capítulo comienza con la presentación de un problema de aplicación no resuelto, referido a los contenidos de ese capítulo. El libro tiene abundantes ejemplos con su solución, ejercicios propuestos sin la respuesta, una autoevaluación con sus respuestas y un glosario de términos al final de cada capítulo.

\subsection{El trabajo de los alumnos}

Los alumnos desarrollaron la experiencia en sus domicilios y en el aula virtual, usando recursos de la tecnología actual. Las clases presenciales estuvieron dedicadas a la enseñanza y al aprendizaje de los temas propios de la materia. "En definitiva, los entornos virtuales de aprendizaje permiten aprender sin coincidir en el espacio ni en el tiempo y asumen las funciones de contexto de aprendizaje que en los sistemas de formación pre- 
sencial desarrolla el aula" (Duart, Sangrá, 2001, p. 31). La llegada masiva de las TIC ha significado para la sociedad una evolución que la educación, como factor de movilidad social, debe acompañar. Freire (2009) señala que "la educación, como proceso basado en conocimiento, comunicación e interacciones sociales se ha visto afectada de forma radical por la emergencia de la cultura digital", la cual a su vez, "ha transformado a sus actores, profesores y estudiantes provocando la necesidad de cambios en las propias instituciones educativas" (Freire, 2009, p. 2). Además, el aula virtual como ámbito de resolución de la actividad favorece el aprendizaje colaborativo. Este aprendizaje en entornos virtuales implica dejar el protagonismo docente y estimular la participación activa, elaborada, profunda, libre de prejuicios, reflexionada entre los alumnos. Esta participación despierta la curiosidad e imaginación, y se presenta como contracara al hecho de que el alumno, en el desarrollo de las tareas en forma virtual, deba enfrentarse solo al estudio, produciéndole una sensación de aislamiento y soledad que puede ser causa de abandono. Esta interacción promueve una construcción social del conocimiento y una visión más amplia del aprendizaje, dejando paulatinamente los enfoques tradicionales y generando verdaderas comunidades de autoconocimiento de acuerdo con Pérez-Mateos (2010).

Los alumnos trabajaron en cada trabajo práctico durante dos semanas. Para facilitarles la lectura y escritura del trabajo, les proporcionamos pautas orientadoras que, como parte de las consignas, acompañan a cada trabajo práctico. Hubo un foro de consultas habilitado para cada trabajo práctico, dedicado a la interacción entre los alumnos, moderado por los docentes. Los docentes utilizaron también el foro para evacuar las dudas planteadas. Las devoluciones a los alumnos de los trabajos evaluados se realizaron a través del espacio de retroalimentación de la plataforma Moodle.

\section{Sobre la evaluación}

La evaluación en la universidad constituye por lo general una parte diferenciada y puntual del proceso educativo. Se implementa en instancias formales planificadas al finalizar unidades temáticas, consistiendo en exámenes parciales y finales, de manera común escritos y separados del proceso de enseñanza y de aprendizaje (Alvarez Méndez, 2003). Desde esta perspectiva, la evaluación cumple la función social e institucional de acreditar saberes y habilidades. Esta acreditación se caracteriza por dos puntos: el primero desvincula el examen del desarrollo de la enseńanza y el aprendizaje, mientras que el segundo desvirtúa la relación del alumno con el docente, sobre todo cuando uno estudia para aprobar y el otro enseña lo que se va a evaluar (Díaz Barriga, 1992).

Las evaluaciones centradas en la medición presuponen que la puntuación en una prueba tiene el mismo significado para todos los individuos y que es "objetiva y neutral". Desde la perspectiva constructivista, nosotros construimos el mundo de acuerdo con nuestros valores y percepciones. El sujeto cognitivo es un constructor activo de sus estructuras de conocimiento. Se evoluciona de un estado cognitivo a otro. Cada uno de los autores que han aportado a esta corriente -como Piaget, Vigotsky, Maturana, Ausubel, Novak, von Glaserfeld, Bruner- tratan de explicar la construcción de ciertas estructuras a partir de otras diferentes. Coherentes con este enfoque, piensan en una "puntuación verdadera", marcando la dificultad de establecer una medición cuantitativa objetiva de los conocimientos del estudiante. Así, tenemos que adherir a Sverdlick (2012) cuando expresa: 
Vicente Messina, Gloria Cittadini y Carlos Pano / La escritura académica y [...] 141

[...] ya nadie dude de que aun definiendo operacionalmente las claves de corrección "la nota" puede diferir si la corrección la efectúan personas diferentes, o incluso la misma persona en diferentes en momentos o días distintos; la mística de la "objetividad" y de la "neutralidad" continúa vigente. [...] En efecto, la lógica positivista que aun prima en la escuela tiene su expresión en la demanda de criterios y parámetros de evaluación 'objetivos', lo que equivale a que sean "neutros" y "verdaderos". Esta idea, asentada en la ilusión de neutralidad o de ecuanimidad de una evaluación "objetiva", supone que existe un valor de verdad por fuera de la construcción que están haciendo los actores en el momento de la evaluación. (Sverdlick, 2012, pp. 150, 151)

De acuerdo con Mateo y Olmo (2008), el presupuesto implícito en lo que se denomina psicometría clásica está en suponer que existe un constructo que explica la naturaleza de la conducta humana, que se puede definir con precisión y por tanto es posible construir una prueba que nos permita capturar su esencia y medirla. Los modelos conductistas dieron soporte teórico a un universo de pruebas denominadas objetivas. Por ello, buscar significados objetivos en las prácticas escolares, sabiendo que el evaluador aporta su subjetividad durante el proceso de evaluación, no es conducente. Esta subjetividad está integrada por supuestos previos acerca del objeto a evaluar y por sus supuestos teóricos e ideológicos. En tal sentido, el evaluador que renuncia a buscar objetividad prefiere el concepto de "intersubjetividad", donde la validación de la información se genera a través de estrategias que permiten a los evaluadores y a los evaluados la posibilidad de acordar o disentir sobre los resultados de la evaluación. En este caso se posee consciencia de que el conocimiento es una construcción social y por lo tanto intersubjetiva, que refleja acuerdos, legitimando así al producto que se obtenga.

\section{1. La evaluación alternativa}

Darling-Hammond (2014) señala que, para muchas personas, la evaluación alternativa se define más facilmente por lo que no es. Cualquier procedimiento que aplique instrumentos cuyo diseño implique un intento de percibir, captar, discernir el rendimiento en forma distinta de las formas clásicas planteadas por la psicometría -es decir, pruebas de elección múltiple o alguna de las pruebas objetivas centrada en la medición y el control de los resultados- se puede considerar como una evaluación alternativa. Desde esta perspectiva, la evaluación alternativa abarca una amplia gama de actividades donde se espera que el alumno construya una respuesta original, en lugar de reconocer una respuesta potencialmente correcta de una lista predeterminada. Las actividades pueden ir desde completar una frase con unas pocas palabras hasta la escritura de un ensayo o el análisis de una investigación de laboratorio donde el alumno participa en forma activa y no sólo como observador.

La evaluación alternativa se presenta como un paradigma de cambio respecto a los modelos de medición, privilegiando la parte integral del proceso enseñanza y de aprendizaje. Por supuesto, las evaluaciones acreditativas pueden usarse para el aprendizaje, pero el interés de la evaluación alternativa está en que la valoración es parte esencial 
del proceso pedagógico. Asimismo, el alumno es participante activo en la construcción de significado, hasta el punto en que su inserción refleja una valoración de las normas de rendimiento y la necesidad de la metacognición en el aprendizaje (Gipps y Stobart, 2003). De acuerdo con Darling-Hammond (2014), el desempeńo de los estudiantes se juzga con criterios pre-establecidos, basados en el discernimiento humano, y apunta a la evaluación de conocimientos complejos y de alto nivel de pensamiento que tengan relación con contextos reales. Se dice que la evaluación alternativa es autentica cuando las tareas que el alumno ejecuta tienen como contexto situaciones del mundo real. Como señalan Mateo y Olmo (2008), cuando aparece un nuevo término, casi al mismo tiempo surgen variaciones en su significado. Este es el caso de la evaluación alternativa, que considera como sinónimos los terminos evaluación alternativa, autentica y de ejecución. La evaluación auténtica y la de ejecución no son lo mismo. Para que una evaluación sea autentica las tareas que deben desarrollar los alumnos deben estar asociadas a la vida real. En la de ejecución, esta condición no es imprescindible.

En definitiva, la evaluación basada en ejecuciones (performance based assessment) y la auténtica son dos modalidades dentro del denominado enfoque alternativo, donde la evaluación de ejecución requiere que los estudiantes, en un momento concreto, justifiquen, construyan, realicen un producto o solución a partir de pautas determinadas (Gips y Stobart, 2003). Siguiendo esta misma línea, Khattri y Sweet (como se cita en Mateo y Olmo, 2008), señalan que adoptar el enfoque de la evaluación de ejecución involucra los siguientes pasos a realizar por los estudiantes: estructurar las tareas objeto de evaluación, aplicar información previa, construir respuestas y explicar el proceso que los ha llevado a una determinada respuesta.

\section{La evaluación de las producciones escritas de los alumnos que participaron en la experiencia}

En la evaluación en los exámenes escritos de matemática, el profesor atiende habitualmente a los razonamientos y a los resultados obtenidos, no reparando en otros aspectos de la escritura.

En nuestra experiencia pretendimos hacer una evaluación más amplia de la escritura académico-matemática. Les planteamos a los estudiantes un trabajo que los responsabilizaba de su propio aprendizaje y buscamos generar hábitos de revisión y control de los propios procesos. La evaluación de estos trabajos necesitó mecanismos que hagan evidentes los distintos aspectos de la escritura académica, además de una participación docente diferente. Esta evaluación obliga a que los docentes encargados deban actuar como co-evaluadores, consensuando no sólo los criterios para dirimir la calidad de un escrito matemático, sino para conocer la destreza del estudiante al momento de comunicar por escrito.

Se trató de evaluar los escritos de los alumnos derivados de la lectura del capítulo antes mencionado, teniendo presente que el tema no fue tratado en las clases presenciales. Los escritos respondieron a las demandas docentes. Estas fueron de dos tipos: 
Vicente Messina , Gloria Cittadini y Carlos Pano / La escritura académica y [...] 143

- Respuestas a preguntas

- Resoluciones de ejercicios

El procedimiento de evaluación consta de los siguientes elementos:

1. Para cada pregunta o ejercicio el equipo docente construyó una respuesta modelo.

2. Hay aspectos a considerar para señalar las fallas, relacionadas con la escritura académica, que los escritos contienen.

3. Hay una escala para clasificar los escritos, cuyas categorías se definen por el grado de afinidad con el modelo construido por el equipo.

4. Las fallas señaladas concurren para clasificar las respuestas con la escala.

5. Los escritos son corregidos por dos docentes y la calificación (clasificación) surge del acuerdo entre ambos.

\section{1. La respuesta modelo}

Para cada pregunta o ejercicio el equipo construyó una respuesta modelo. Esta respuesta será obviamente correcta, estará convenientemente fundamentada, ordenada en su presentación y explicada.

Los aspectos considerados y acordados por el grupo de investigación para la evaluación de los escritos son:

- Argumentación

- Cierre

- Coherencia

- Cohesión

- Gramática

- Presentación

- Semática

- Tema

- Representación

En el proceso de corrección nos interesa detectar las fallas que el estudiante-escritor comete en los aspectos señalados. Para eso, a continuación describimos las fallas a considerar en los distintos aspectos:

Argumentación: el escrito a evaluar contiene razonamientos inadecuados, desarrolla demostraciones incorrectas, formula argumentos inapropiados, carece de explicaciones.

Cierre: en el escrito no se definen ni explican símbolos o términos que son utilizados en el trabajo.

Coherencia: en el escrito faltan títulos o subtítulos cuando debieran estar. Se omite la transcripción de la pregunta o del enunciado del ejercicio. No se señala la o las respuestas. Falta la presencia de un hilo conductor que le otorgue unidad al texto producido. 
144/ Perspectivas Metodológicas /19/Vol. II /Año 2017

Cohesión: el escrito presenta partes desconectadas, no tiene conexión lógica entre algunos enunciados ni entre párrafos verbales y gráficos o tablas. Carece de conectores adecuados.

Gramática: el escrito contiene faltas de ortografía, oraciones mal construidas o mal enlazadas, o hace un empleo incorrecto de los signos de puntuación.

Presentación: el trabajo debe respetar las normas de presentación: título, subtítulo, apellido y nombre, curso al que pertenece, fecha de entrega, tipo y tamaño de letra.

Semántica: el escrito presenta un uso incorrecto de los términos, en particular de los términos técnicos o confude el significado de las palabras.

Tema: el escrito no responde a la pregunta o desacuerda con el enunciado del ejercicio.

Representación: el escrito omite nombres globales en las tablas de datos y gráficos, y en sus partes constitutivas. Exhibe un tratamiento inadecuado de las expresiones algebraicas o las locuciones simbólicas.

\subsection{La escala}

Para evaluar las respuestas de los alumnos usamos una escala ordinal. Las clases de la escala son:

- respuesta correcta

- respuesta próxima a la correcta

- respuesta próxima a la incorrecta

- respuesta incorrecta

- respuesta en blanco

Se asignó a cada respuesta un valor numérico a los fines de conformar una nota para cada ejercicio, como se indica en la tabla $\mathrm{N}^{\circ} 1$.

\begin{tabular}{|l|c|}
\hline \multicolumn{1}{|c|}{ Respuesta } & Nota \\
\hline Respuesta correcta & $\mathbf{4}$ \\
\hline Respuesta próxima a la correcta & $\mathbf{3}$ \\
\hline Respuesta próxima a la incorrecta & $\mathbf{2}$ \\
\hline Respuesta incorrecta & $\mathbf{1}$ \\
\hline Respuesta en blanco & $\mathbf{0}$ \\
\hline
\end{tabular}

Tabla $\mathbf{N}^{\circ}$ 1. Asignaciones de valores numéricos a las respuestas

\section{Consideramos:}

- correcta toda respuesta que guarda relación de semejanza con la respuesta modelo.

- próxima a la correcta a una respuesta con más partes afines al modelo correcto que partes contrarias al mismo. 
Vicente Messina, Gloria Cittadini y Carlos Pano / La escritura académica y [...] 145

- próxima a la incorrecta a una respuesta con menos partes afines al modelo correcto que partes contrarias al mismo.

- incorrecta, a una respuesta que, en todas sus partes, resulte contraria al modelo.

- respuesta en blanco en el caso que no haya respuesta.

Si bien los resultados a que lleguen los estudiantes hacen, desde el punto de vista de la matemática, a la calidad de los escritos, no son determinantes para la clasificación. Un resultado matemáticamente incorrecto no será clasificado como 1 (respuesta incorrecta) si no hay fallas en los aspectos considerados o las hay en una cantidad no significativa. De manera similar, un resultado matemáticamente correcto no será clasificado como 4 (respuesta correcta) si hay fallas en los aspectos considerados en una cantidad significativa.

\subsection{La corrección de los escritos}

De los escritos de los alumnos que participaron de la experiencia elegimos ocho al azar. Las condiciones materiales en que se realizó la experiencia no nos permitieron considerar, para este artículo, la totalidad. Todos los escritos surgieron a partir de la lectura del capítulo del texto indicado más arriba.

Cada escrito es corregido por dos docentes. Si ambos coinciden en la asignación de la clase de la escala, esta queda como definitiva; en caso contrario, interactuarán para resolver por consenso, con convencimiento mutuo, la asignación.

La lectura y evaluación de las producciones de los alumnos están afectadas por las concepciones que el docente-investigador tiene sobre la evaluación y estas son puestas en juego en la tarea. El procedimiento de evaluación de los escritos, por parejas docentes participantes del proyecto, con criterios establecidos y con respuestas modelo que utilizamos en la experiencia, hace que ese equipo deba discutir, reflexionar, analizar, acordar. Es un procedimiento que no busca la objetividad, pero como se obligan a consensuar mediante el análisis y la reflexión conjunta, a la luz de los criterios establecidos, la calificación que se consiga será una construcción intersubjetiva y por lo tanto con legitimidad.

\subsection{Definición de la matriz de fallas}

La matriz de un alumno da cuenta de las fallas que surgen en los distintos aspectos de cada ejercicio. Hay nueve aspectos a considerar y quince ejercicios en la totalidad de los trabajos prácticos.

Cuando un alumno en el ejercicio i-ésimo incurre en por lo menos una falla del aspecto j-ésimo lo indicamos con 1 (uno) y lo denotamos éxito, caso contrario lo indicamos con 0 (cero) y lo señalamos como fracaso. Esta información para cada alumno define una matriz de quince filas por nueve columnas. Es una matriz de unos y ceros, con lo cual se tienen así 135 variables dicotómicas. 
En la tabla $\mathrm{N}^{\circ} 2$ presentamos la matriz correspondiente al alumno A. Habiendo agregado una columna con la nota arriba definida y una fila con la suma por columnas que denominamos vector de éxitos por aspectos.

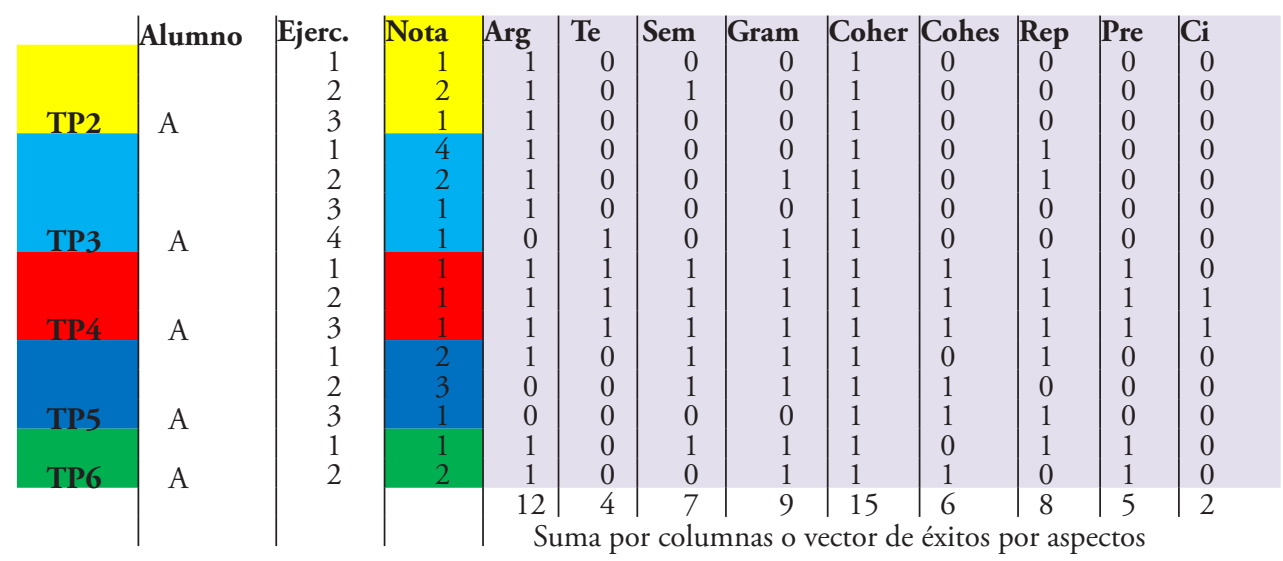

Tabla No2. Matriz de fallas del alumno A

Es posible mostrar la evolución de cada alumno en sus escritos, a través de la variable nota transformada, en función de la sucesión temporal de trabajos prácticos. La nota transformada estará constituida por cada una de las notas obtenidas en cada ejercicio del trabajo práctico correspondiente. Para calcular el valor asignado a la variable se sumaron las notas obtenidas por el alumno en cada ejercicio del trabajo práctico y esa suma se dividió por cuatro veces la cantidad de ejercicios del trabajo práctico. Así, la nota transformada queda independiente de la cantidad de ejercicios del trabajo práctico. Hallamos la nota transformada para los ocho alumnos y calculamos el coeficiente de correlación lineal, entre el número de trabajo práctico (indicador del tiempo) y la nota transformada para cada alumno, obteniendo los valores mostrados en la tabla $\mathrm{N}^{\circ} 3$.

\begin{tabular}{|l|c|c|c|c|c|c|}
\hline & TP2 & TP3 & TP4 & TP5 & TP6 & $\begin{array}{l}\text { Coeficiente de } \\
\text { correlación lineal R }\end{array}$ \\
\hline Alumno A & 0,33 & 0,50 & 0,25 & 0,50 & 0,38 & 0,1212 \\
\hline Alumno B & 0,75 & 0,44 & 0,58 & 0,67 & 0,88 & 0,4574 \\
\hline Alumno C & 0,67 & 0,63 & 0,75 & 0,92 & 0,50 & 0,6481 \\
\hline Alumno D & 0,75 & 0,81 & 0,58 & 0,92 & 0,88 & 0,4293 \\
\hline Alumno E & 0,67 & 0,44 & 0,75 & 0,67 & 0,88 & 0,6392 \\
\hline Alumno F & 0,42 & 0,50 & 0,25 & 0,33 & 0,33 & 0,0000 \\
\hline Alumno G & 0,67 & 0,5 & 0,58 & 0,42 & 0,88 & 0,2998 \\
\hline Alumno H & 0,33 & 0,69 & 0,42 & 0,50 & 0,50 & 0,4004 \\
\hline
\end{tabular}

Tabla $N^{\circ} 3$. Coeficiente de correlación lineal para cada uno de los ocho alumnos

Se puede observar que los coeficientes de correlación lineal son no negativos. Esto indica que en el transcurso de la experiencia la nota transformada fue en aumento. Puede considerarse que la evolución de los escritos académico-matemáticos de los alumnos fue 
Vicente Messina , Gloria Cittadini y Carlos Pano / La escritura académica y [...] 147 mejorando en los sucesivos trabajos prácticos de la experiencia. Otro indicador que nos permite apreciar la dispersión de la distribución de éxitos de un alumno en los aspectos es el concepto de entropía.

\section{5. Entropía}

En Garcia Mayoraz (1989), leemos que entropía es un término de origen griego, viene de la palabra tropee, que significa transformación o cambio. Desde el punto de vista termodinámico, es una medida de la transformación de la energía. Fue creado en 1876 por el físico alemán Rudolf Claussius (1822-1888). Permite cuantificar los valores de transformación de la energía, calculando las variaciones de entropía entre dos estados de un sistema que evoluciona entre ellos. Ludwig Boltzmann (1844-1906) logró llegar al concepto de entropía partir de la teoría atómica y la probabilidad, preguntándose sobre los factores que afectan a la probabilidad de que una molécula se mueva con cierta velocidad. Según su teoría, un sistema que evoluciona espontáneamente de un estado de menor probabilidad a otro de mayor probabilidad, es decir, mayor desorden, mayor entropía y el estado más desordenado es el estado más probable. A finales de la década del cuarenta, Claude E. Shannon (1916-2001) propone una teoría que mide la cantidad de información contenida en un mensaje. Se puede calcular un valor promedio de información como la cantidad media de información por símbolo de la fuente, expresándose como $\mathrm{H}$.

$$
\mathrm{H}=-\mathrm{p}_{1} \ln \mathrm{p}_{1}-\mathrm{p}_{2} \ln \mathrm{p}_{2}-\ldots-\mathrm{p}_{\mathrm{k}} \ln \mathrm{p}_{\mathrm{k}}
$$

En una distribución de frecuencias, la entropía se calcula con la fórmula anterior reemplazando las probabilidades $\mathrm{p}_{\mathrm{i}}$ por las frecuencias relativas correspondientes. De acuerdo con Reuchlin (1976), esta noción de dispersión de una distribución nominal podrá ser mayor cuanto mayor es el número de clases. Para un número fijo de clases, será mayor si las observaciones se reparten igualmente sobre todas las clases en lugar de concentrarse sólo en algunas. Se puede explicitar y cuantificar esta noción definiendo y calculando la entropía de la distribución de éxitos en los aspectos. La entropía para los ocho alumnos se muestra en la tabla $\mathrm{N}^{\circ} 4$.

\begin{tabular}{|l|l|l|l|}
\hline Alumno A: 2,07 & Alumno B: 1,74 & Alumno C: 1,90 & Alumno D: 1,89 \\
\hline Alumno E: 2,00 & Alumno F: 1,87 & Alumno G: 1,72 & Alumno H: 1,90 \\
\hline
\end{tabular}

Tabla $N^{\circ}$ 4: Valores de entropía de los escritos de los ocho alumnos

Se observa que el alumno G, que obtuvo menor entropía, tiene una mayor concentración de éxitos en algunos de los aspectos, y que el alumno A, con la máxima entropía, tiene éxitos repartidos entre todos los aspectos. Con lo cual podemos concluir que al alumno $\mathrm{G}$ es conveniente que revise los aspectos donde se concentran el mayor número de éxitos. En cambio al alumno A será necesario que ponga atención en todos los aspectos. En el gráfico $\mathrm{N}^{\circ} 1$ se muestra las distribuciones de éxito estos dos alumnos. El alumno A tiene en sus escritos más éxitos que el alumno G. Se puede observar mayor dispersión en A que en G. 


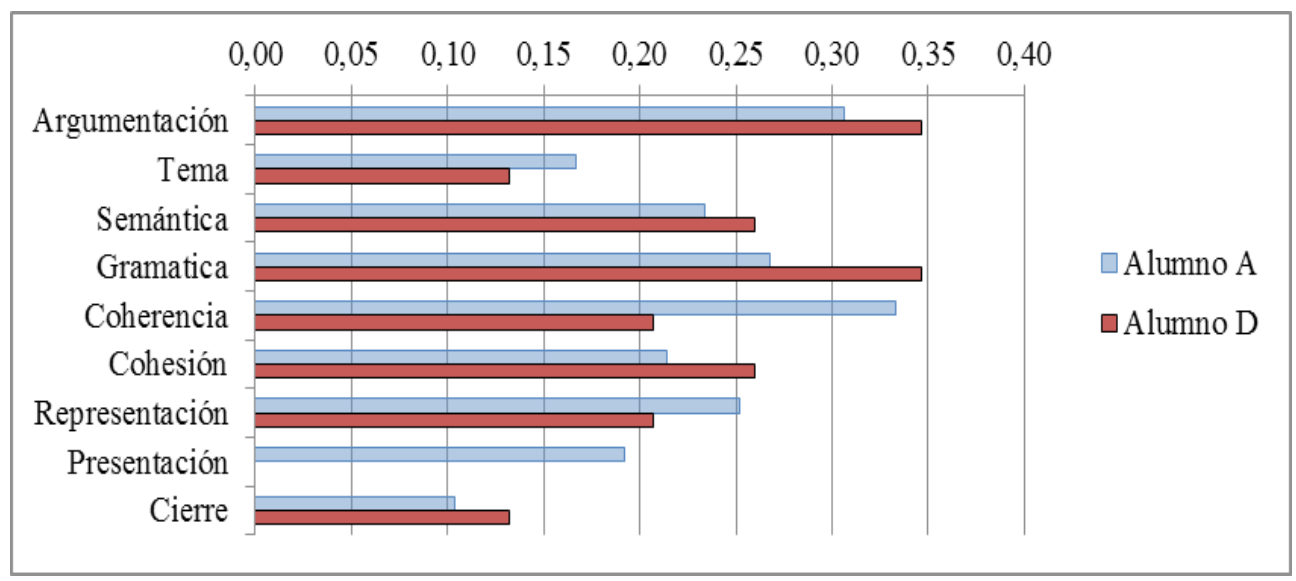

Gráfico $\mathrm{N}^{\circ}$ 1: Dispersión de éxitos, por aspectos, en dos alumnos

\section{Reflexiones finales}

Este estudio, llevado a cabo a principios del año 2015, permitió observar los escritos de alumnos de ingeniería que recién comenzaban su formación. Nos encontramos con escritos que muestran cuáles alumnos tienen dificultas para comunicar sus ideas. Nos consta el poco uso que hacen los estudiantes de los textos recomendados. Por eso, entendemos que, como docentes universitarios, debemos ocuparnos de la escritura académica, proponiendo actividades dirigidas a su práctica. El objetivo de la experiencia consistió en poner en función una actividad de alfabetización académica para propender al mejoramiento del aprendizaje de las secciones cónicas. En la experiencia que contamos, los alumnos autónomamente leyeron y escribieron, siguiendo determinadas pautas, sobre un tema de la geometría analítica que deben dominar. Habremos de generar actividades de escritura de textos académicos en álgebra lineal y geometría analítica que abran caminos al aprendizaje sólido en la compleja red de conceptos que los constituyen y que preparen a los estudiantes para poder profundizarlos y aplicarlos. Sostenemos que los estudiantes alfabetizados académicamente serán ingenieros capaces de enfrentar con solvencia los desafíos de la vida profesional y adaptarse con comodidad a los cambios sociales y tecnológicos que ocurren con una rapidez cada vez mayor.

\section{Bibliografía}

— Álvarez Méndez, J. M. (2003). La evaluación a examen. Ensayos críticos. Buenos Aires: Miño y Dávila.

- Bruning, R., Schraw, G., Norby, M. y Ronning, R. (2005). Psicología cognitiva y de la instrucción (4ta. ed.). Madrid: Pearson Prentice Hall.

— Carlino, P. (2005). Escribir, leer y aprender en la universidad. Una introducción a la 
Vicente Messina , Gloria Cittadini y Carlos Pano / La escritura académica y [...] 149 Alfabetización Académica. Buenos Aires: Fondo de Cultura Económica de Argentina.

- Castedo, M. L. (1995). "Construcción de lectores y escritores". Lectura y vida, 16(3), pp. 5-24.

- Darling-Hammond, L. (2014). Next generation assessment. Moving beyond the bubble test to support $21^{\text {st }}$ century learning. San Francisco: John Wiley and Sons.

- Díaz Barriga, A. (1992). Didáctica. Aportes para una polémica. Buenos Aires: Aique.

- Doyle, W. (1981). "La investigación sobre el contexto del aula: hacia un conocimiento básico para la práctica y política de formación del profesorado”. Revista de Educación, 277, pp. 29-42. Recuperado el 07/04/2016 de http://www.mecd. gob.es/dctm/revista-de-educacion/articulosre277/re2770200503.pdf?documentI$\mathrm{d}=0901 \mathrm{e} 72 \mathrm{~b} 813 \mathrm{c} 3054$

- Doyle, W. (1982). Academic work. National Commission on Excellence in Education. Washington: Department of Education. Recuperado el 09/05/2016 de la base de datos ERIC.

- Duart, J.M. y Sangrá, A. (2001). Aprender en la virtualidad. Barcelona: Gedisa.

- Freire, J. (2009). Monográfico "Cultura digital prácticas creativas en educación". Revista de Universidad y Sociedad del Conocimiento. Recuperado el 10/06/2015 de http://www.raco.cat/index.php/Rusc/article/viewFile/129749/179184

- Garcia Mayoraz, J. (1989). Entropía y Lenguajes. Buenos Aires: Hachette.

- Gipps, C. y Stobart, G. (2003). Alternative Evaluation. En Kellaghan, T. y Stufflebean, D. (Eds.). International Handbook of Educational Evaluation (pp. 549-573). Dordrecht, The Netherlands: Kluwer Academic Publishers.

- Kozak, A. M., Pastorelli, S. y Vardanega, P. (2007). Nociones de geometría analítica y álgebra lineal. Buenos Aires: McGraw-Hill Interamericana.

- Mateo, J. y Olmo, F.M. (2008). La evaluación alternativa de los aprendizajes. Barcelona: Ediciones Octaedro.

- Pérez-Mateo Subirà, M. (2010). La dimensión social en el proceso de aprendizaje colaborativo virtual: El caso de la UOC. Comprender y abordar la dimensión social en el trabajo en grupo virtual. Universitat Oberta de Catalunya. Recuperado de http://www.tdx.cat/bitstream/handle/10803/37113/tesi_mperezmateo1.pdf;jsessionid=F5DDD10BA07494D7DE1095F43B2218EB.tdx1? sequence $=1$. Recuperado el 01/03/2015.

— Pano, C., Cittadini, G., Pustilnik, I., Sara, A. y Messina, V. (2013). Alfabetización académica en entornos virtuales de aprendizaje y evaluación innovadora con función pedagógica. Proyecto con asiento en Universidad Tecnológica Nacional Regional Buenos Aires, reconocido por el código 25/C138 en el ámbito del Programa de Incentivos a los Docentes Investigadores.

— Reuchlin, M. (1976). Compendio de estadística: Método conceptual. Madrid: Pablo del Rio Editores.

- Steiman, J. (2008). Más didáctica (en la educación superior). Buenos Aires: UNSAM Edita.

— Sverdlick, I. (2012). ¿Qué hay de nuevo en evaluación educativa? Políticas y prácticas en la evaluación de docentes y alumnos. Propuestas y experiencias de autoevaluación. Noveduc: Buenos Aires.

— Thaiss, C., Bräuer, G., Carlino, P., Ganobcsik-Williams, L. y Sinha, A. (2012). Writing programs worldwide. Profiles of academic writing in many places. Recuperado de http://wac.colostate.edu/books/wpww/ 